University of Nebraska - Lincoln

DigitalCommons@University of Nebraska - Lincoln

Virology Papers

Virology, Nebraska Center for

December 2001

\title{
Interferon regulatory factor 7: a key cellular mediator of LMP-1 in EBV latency and transformation
}

Luwen Zhang

University of Nebraska-Lincoln, Izhang2@unl.edu

Joseph S. Pagano

University of North Carolina, Chapel Hill, NC

Follow this and additional works at: https://digitalcommons.unl.edu/virologypub

Part of the Virology Commons

Zhang, Luwen and Pagano, Joseph S., "Interferon regulatory factor 7: a key cellular mediator of LMP-1 in EBV latency and transformation" (2001). Virology Papers. 27.

https://digitalcommons.unl.edu/virologypub/27

This Article is brought to you for free and open access by the Virology, Nebraska Center for at DigitalCommons@University of Nebraska - Lincoln. It has been accepted for inclusion in Virology Papers by an authorized administrator of DigitalCommons@University of Nebraska - Lincoln. 


\title{
Interferon regulatory factor 7: a key cellular mediator of LMP-1 in EBV latency and transformation
}

\author{
Luwen Zhang ${ }^{1,2, *}$ and Joseph S. Pagano ${ }^{1,2,3}$ \\ ${ }^{1}$ Lineberger Comprehensive Cancer Center, ${ }^{2}$ Department of Microbiology and Immunology, \\ ${ }^{3}$ Department of Medicine; University of North Carolina, Chapel Hill, NC 27599-7295, USA \\ *Corresponding author.
}

\begin{abstract}
Interferon regulatory factor 7 (IRF-7) was cloned within the biological context of Epstein-Barr virus (EBV) latency, and has an intimate relation with EBV. EBV latent membrane protein 1 (LMP-1) regulates IRF-7 both by inducing the expression of IRF-7 and by activating IRF-7 protein through phosphorylation and nuclear translocation in a post-translational manner. The activated IRF-7 then functions to regulate both EBV and cellular target genes involved in latency, transformation and immune regulation. IRF-7 appears to be a key cellular latency protein involved in both the pathogenesis and persistence of EBV infection.
\end{abstract}

Keywords: IRF-7, LMP-1, EBV, latency

\section{Introduction}

Epstein-Barr virus (EBV) is a human herpes virus of increasing medical importance. EBV infection has been associated with the development of nasopharyngeal carcinoma (NPC) and Burkitt's lymphoma (BL). In addition, EBV infection is an important cause of lymphomas in severely immunocompromised people, especially patients with AIDS and organ-transplant recipients. EBV infection can significantly affect cell growth, differentiation and death by regulating complex genetic regulatory networks. Mounting evidence indicates that EBV can usurp cellular molecules for its own functions and persistence in the host in adaptations obviously acquired through evolution of virus-host interactions. ${ }^{1,2}$

Historically, interferon regulatory factor (IRF) was named after the cloning of IRF-1, which was implicated as a regulator of an interferon (IFN) gene at that time. ${ }^{3}$
Currently, the IRF family has a total of nine members with multiple functions. ${ }^{4,5}$ The hallmark of this family is its N-terminal DNA-binding domain (DBD), which has five well-spaced, conserved tryptophan repeat sequences. The tryptophan repeat region can form a helix-turn-helix motif that latches onto DNA. ${ }^{6}$ Because of the conserved DBDs, IRFs can bind to consensus or similar DNA sequences with different names: interferon stimulated response element (ISRE), positive regulatory element (PDE), and interferon consensus sequence (ICS), all of which have conserved GAAA repeats. ${ }^{7-11}$ The $\mathrm{C}$-terminal region of IRFs is variable and defines multiple biologic functions including regulation of cell cycle, oncogenesis, apoptosis, and immune responses. In addition, IRF members interact with one another and with other transcription factors, such as Stats and PU.1, and as a consequence greatly expand the effects of IRFs., ${ }^{4,5}$

IRF-7 was cloned and identified within the biological context of EBV latency, based on its binding activity to the BamHI Q promoter (Qp) used in latent EBV infection for transcription of EBNA-1. ${ }^{12}$ The cDNA library selected for cloning IRF-7 was constructed from EBV-transformed human B lymphocytes, in which expression of IRF-7 was later found to be high. ${ }^{12,13}$ Serendipity thus led to the discovery of IRF-7 and helped in its cloning. Although a low level of expression has been detected in all tissues examined, IRF-7 is a lymphoid-specific factor that is predominantly expressed in the spleen, thymus, and primary blood lymphocytes (PBL). Tissue-specific splicing is apparent: there are two major forms $(2.0$ and $2.6 \mathrm{~kb})$ in spleen and thymus, and only one $(2.0 \mathrm{~kb})$ in PBL. ${ }^{12}$ Multiple splicing variants of IRF-7 have been identified, which are named 


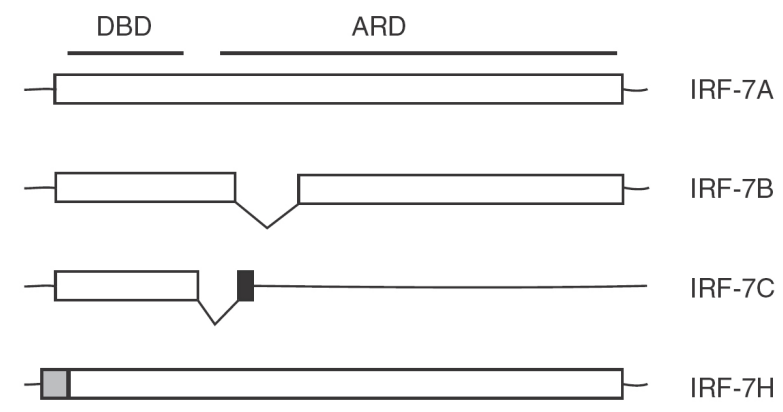

Figure 1. Structure of IRF-7 protein. ORFs are represented by open and solid bars. IRF-7B is missing 29 amino acids in the middle. IRF-7C has a 13-amino acid sequence difference from IRF-7A in its C-terminus, indicated by the solid bar. IRF-7H differs from IRF-7A by 18 amino acids at the very N-terminal region indicated by the gray bar. DBD and ARD are also indicated.

IRF-7A, 7B, 7C, and 7H. ${ }^{12,14}$ At least in PBL, IRF-7A appears to be the major protein form. As expected, IRF-7 binds to ISRE as well as PDE, ${ }^{12,14,15}$ and the DBD is localized in the N-terminal region of the protein. ${ }^{12}$ Through random selection, the consensus sequence for IRF-7 binding sites was identified: 5' GAAA/TNC/TGAAANT/C$3^{\prime} .{ }^{16}$ The C-terminal region of IRF-7 contains the activation and regulation domain (ARD) (Figure 1); multiple subdomains in the region have been proposed. ${ }^{14,17}$

The effects of IRF-7 are not limited to EBV. Since IRF-7 was cloned, extensive work has been done in both EBV latency and in virally induced IFN systems to define the biological functions of IRF-7. A number of papers indicate that IRF-7 plays a pivotal role in the induction of IFN genes after viral infections. ${ }^{14,15,17-19}$ The biological functions and regulation of IRF-7 in EBV latency are still to be worked out. In this paper, we will focus mainly on two aspects of IRF-7 related to EBV latency: (1) how EBV regulates IRF-7; and (2) how IRF-7 regulates target genes involved in EBV latency, transformation and immune regulation.

\section{Regulation of IRF-7 in EBV latent infection states}

\section{LMP-1 induces the expression of IRF-7}

The biologic hallmark of the EBV-cell interaction is latency. Several types of latency have been described, each having its own distinct pattern of gene expression. In type I latency, EBV nuclear antigen 1 (EBNA-
1) is the major, and possibly the only, viral protein expressed. However, in type III latency, nine viral proteins are expressed, including latent membrane protein 1 (LMP-1). ${ }^{1,2}$

LMP-1 is an integral membrane protein that is expressed during EBV latency. LMP-1 acts as a constitutively active receptor-like molecule that does not need the binding of a ligand. ${ }^{2,20}$ LMP-1 has six transmembrane-spanning domains with a long C-terminal domain, which is located in the cytoplasm. ${ }^{21}$ The transmembrane domains mediate oligomerization of LMP-1 molecules in the plasma membrane, a prerequisite for LMP-1 functions. ${ }^{20,22}$ LMP-1 can activate many important cellular signaling molecules including tumor necrosis factor receptor (TNFR)-associated factors (TRAFs), TNFR-associated death domain protein (TRADD), nuclear factor $\kappa B$ (NFKB), c-Jun N-terminal kinase (JNK), janus kinase 3 (JAK3) as well as signal transducer and activator of transcription 1 (STAT 1). ${ }^{2}$ Through activation of these cellular molecules, LMP-1 is emerging as a central effector of altered cell growth and survival in EBV immortalization and transformation processes, as well as the effector of the adhesive and invasive potential of EBV-transformed cells. $^{23-28}$

The intimate relation between IRF-7 and EBV latency was first noticed after the initial cloning. IRF-7 protein is expressed at high levels in EBV type III latency, in which LMP-1 is expressed; however, IRF-7 is barely expressed in type I latency, in which LMP- 1 is not expressed. This result suggested that one or more viral proteins expressed in type III latency might be responsible for the induction of IRF-7. In order to narrow down the potential responsible gene(s), cells infected with EBV P3HR-1 or B958 strains were used to examine the expression of IRF-7. ${ }^{29}$ One difference between the P3HR1 and B95-8 strains is that cells infected with the B95-8 strain express high levels of LMP-1. In contrast cells infected with P3HR1 virus express very low levels of LMP-1 and low levels of IRF-7. ${ }^{28,30}$ High levels of IRF-7 are associated with LMP1 expression in B95-8-infected cell lines. ${ }^{30}$ Furthermore, stable expression of LMP-1 in EBV-negative B cells increased the expression of endogenous IRF-7. Also, transient transfection of LMP-1 into B cells could induce the expression of endogenous IRF-7. ${ }^{30}$ All these data suggest that LMP-1 is a necessary factor for the stimulation of the expression of IRF-7 in EBV-infected cells.

The mechanism for the induction of IRF-7 RNA by LMP-1 is partially elucidated. LMP-1 is a membrane protein and depends heavily, if not exclusively, on cellular 
signaling molecules for its function. Generally, two regions in the C-terminus of LMP-1 have been shown to initiate signaling processes, the C-terminal activator regions 1 (CTAR-1) and 2 (CTAR-2). Our results provide strong evidence that the two regions of LMP-1 can independently induce the expression of IRF-7. The important implication of this result is that IRF-7 may be an essential factor for EBV latency, and LMP-1 may need to use two independent pathways, regardless of different cellular environments, to ensure the induction of IRF-7. Specifically, TRAFs and NFKB are involved in the induction of IRF-7 expression. ${ }^{31}$

\section{LMP-1 activates IRF-7 protein in a post-translational manner}

In addition to induction of the expression of IRF7, LMP-1 can also regulate IRF-7 protein activity posttranslationally. Originally, we found that both IRF-7 and LMP-1 are required for the activation of endogenous cellular transporter associated with antigen processing 2 (Tap-2). ${ }^{32}$ To understand the apparent synergy between LMP-1 and IRF-7, we tested the ability of LMP-1 to regulate IRF-7 by phosphorylation and nuclear translocation because post-translational modification of IRF-7 had been reported. ${ }^{14,15}$ Also, regulation of cellular genes by phosphorylation and nuclear translocation is not without precedent for LMP-1. It is well established that LMP-1 phosphorylates I $\mathrm{B}$ and translocates NF- $\kappa \mathrm{B}$ from the cytoplasm to the nucleus. ${ }^{1,2}$

Interestingly, IRF-7 itself is a phosphoprotein. However, the expression of LMP-1 augments the phosphorylation status of IRF-7. Furthermore, IRF-7 itself is predominantly a cytoplasmic protein, and the expression of LMP-1 facilitates its movement to the nucleus. ${ }^{32}$ These results suggest that endogenous IRF-7 is constitutively activated in EBV latency where both IRF-7 and LMP-1 are expressed. As expected, IRF-7 in EBV type III latency is indeed constitutively active as judged by phosphorylation status and nuclear localization. ${ }^{32}$

The mechanism for the post-translational activation of IRF-7 protein by LMP-1 is completely unknown. Other than EBV LMP-1, other agents such as viral infection (e.g. Sendai virus) and DNA damaging agents (e.g. UV light) can activate IRF-7 protein by phosphorylation and nuclear translocation. ${ }^{14,15,17,33}$ It seems likely that the activation of IRF-7 protein by phosphorylation and nuclear translocation is a common theme for the regulation of IRF-7 activity.

\section{IRF-7 regulates EBV latency}

\section{Regulation of an EBV latency promoter by IRF-7}

EBNA-1 is the sole viral protein needed for the replication of the EBV episome and maintenance of the latent infection state; both events are essential for viral persistence and cell transformation. Qp is used for the transcription of EBNA-1 mRNA in type I latency; however, Qp is silent in type III latency, and another promoter $(\mathrm{C} / \mathrm{Wp})$ is used for EBNA-1.,

The functional importance of Qp in the EBV life cycle is underscored by the following facts: (1) Qp usage is not only related to EBV persistence in an immunocompetent host, but it is also associated with several EBV-positive tumors; (2) all EBV-positive tumor specimens collected from Africa, North America, and Asia have conserved Qp sequence; ${ }^{34}$ and (3) conserved structural and functional cis-elements (e.g. ISRE and the Q-locus, Figure 3) also exist in the old world primate lymphocryptoviruses that are simian EBV-like viruses. ${ }^{35}$ Thus it is obvious that dissecting the regulation of Qp is essential for understanding the viral program in persistence and pathogenesis. Both EBNA-1 and host factors are involved in the transcriptional regulation of Qp. EBNA-1 can bind to downstream elements of $\mathrm{Qp}$, the Q locus, and acts in an autoregulatory manner to repress Qp transcription. ${ }^{36,37}$ However, E2F-1 overcomes EBNA-1-mediated repression of Qp in transient transfection assays, and E2F-1 binds to the Q locus and displaces the binding of EBNA- $1,{ }^{38}$ so that the promoter is regulated in a cell-cycle dependent manner with the peak level of EBNA-1 mRNA reached synchronously with S-phase. ${ }^{39}$

There is a body of evidence to support the role of IRF7 as a negative regulator of Qp. IRF-7 was cloned from its binding activity to $\mathrm{Qp}$ and subsequently proven to bind to Qp both in vitro with EMSA and in vivo with chromosomal immunoprecipitation (CHIP) assay as used in the analysis of IRF-7 binding to the Tap-2 promoter $^{12,32}$ (also Zhang and Pagano, unpublished results). The expression of IRF-7 is high in EBV type III latency, where Qp is inactive, and is low in EBV type I latency, where Qp is active. Qp reactivation from type III latency by cell fusion is associated with a sharp reduction in expression of IRF$7 .{ }^{40}$ IRF-7 represses the activity of Qp-reporter constructs in transient transfection assays. Finally, IFN- $\alpha$, but not IFN- $\gamma$, is able to induce the expression of IRF-7, ${ }^{14,30,41}$ and IFN- $\alpha$, but not IFN- $\gamma$, represses endogenous Qp activity in type I cells, as well as the activity of Qp-reporter 


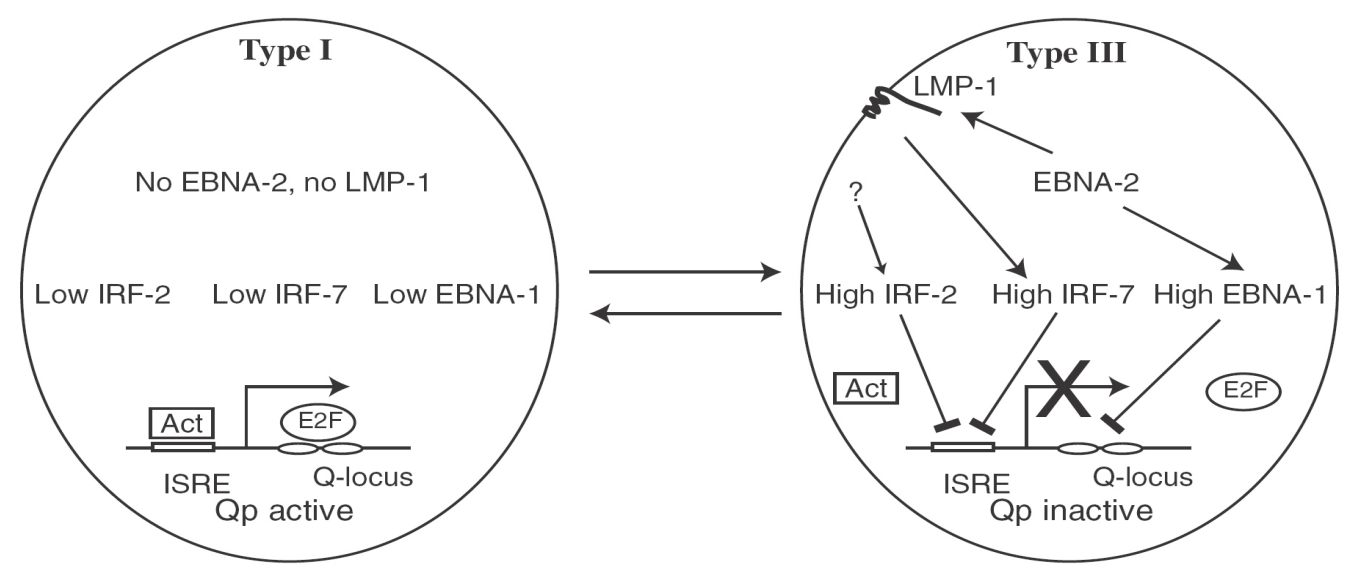

Figure 2. Model for the inactivation of Qp in type III latency. Schematic diagram of type I and type III latency cells and their Qp status is depicted. In type I latency, there is neither LMP-1 nor EBNA-2 expression, and only low level expression of IRF7, IRF-2, and EBNA-1. The putative activator(s) of Qp (Act) functions through ISRE and E2F functions through the Q locus to activate Qp. However in type III latency, EBNA-2 induces the expression of LMP-1, which in turn causes high expression of IRF-7. EBNA-2 also increases the expression of EBNA-1. How IRF-2 is up-regulated is currently unknown. The high levels of IRF-7, IRF-2 and EBNA-1 collectively inactivate Qp in type III latency by keeping the putative activator, as well as E2F, away from Qp.

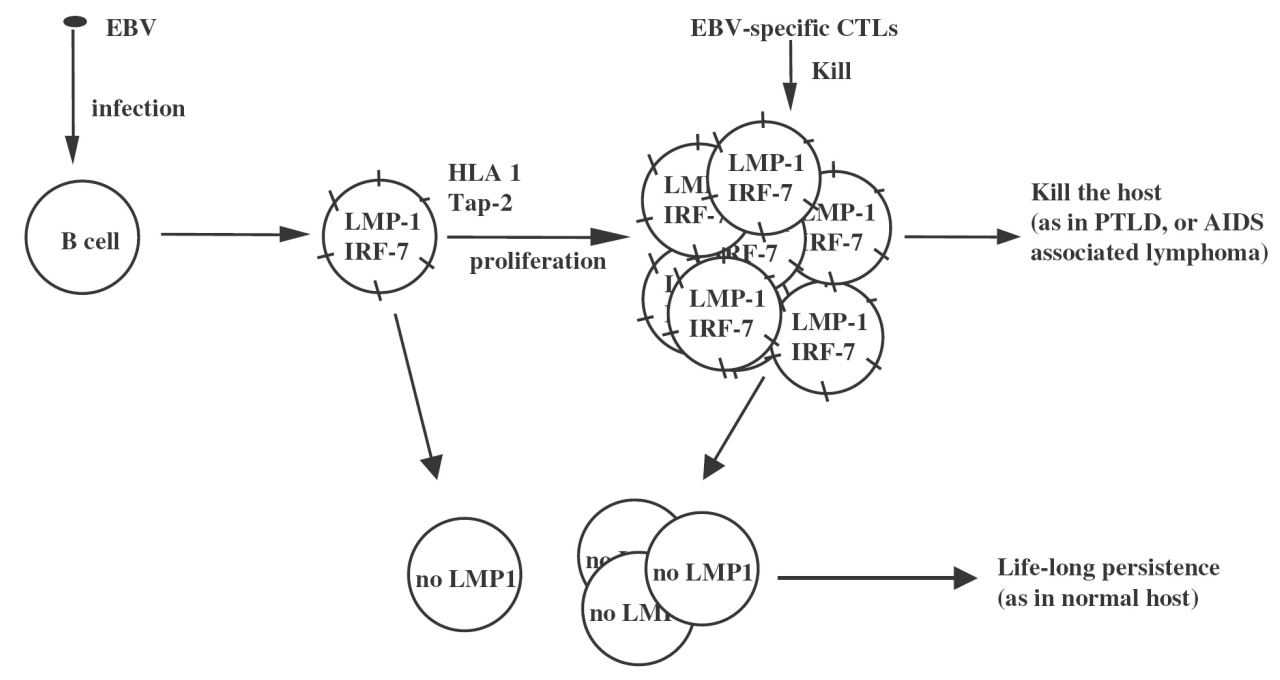

CTLs do not recognize

Figure 3. Schematic diagram of EBV-cell interactions underlying primary infection and persistence. First in acutely infected B cells, EBV expresses all the viral latency genes, including LMP-1, which in turn causes higher levels of expression of IRF-7, Tap-2, and HLA class I molecules. These cells proliferate transiently before the EBV-specific CTLs develop. Second, a life-long viral persistent state with no LMP-1 expression arises, which has selectively survived EBV-specific CTL attack; these cells escape immune surveillance. A presumed latency switch generates the persistent state. 
constructs in transient transfection assays. ${ }^{30}$ Thus, the data overwhelmingly support the idea that IRF-7 is a repressor of Qp, functioning to keep the promoter inactive in type III latency, where $\mathrm{C} / \mathrm{Wp}$ is used instead.

The fact that LMP-1 induces the expression of IRF-7 predicts that LMP-1 may be able to repress Qp through IRF-7. Indeed, LMP-1 could repress the activity of Qp reporter constructs and the endogenous Qp. The repression of Qp is apparently associated with the ability to induce the expression of endogenous IRF-7. EBNA-2, which could not stimulate the expression of IRF-7, could not repress the activity of Qp reporter constructs. Nor did LMP1 repress Qp reporter and endogenous Qp in Akata cells in which IRF-7 could not be induced. Furthermore, mutations in the ISRE of Qp abolish its repression by LMP1 or IRF-7. ${ }^{30}$

It is apparent that Qp activity is a balanced outcome of positive and negative regulators, with IRFs strongly implicated in its regulation. In addition to IRF-7, IRF-2 also negatively regulates $\mathrm{Qp}$, and its expression is associated with EBV type III latency. ${ }^{40}$ Thus in type III latency, Qp may be turned off by a combination of LMP-1, IRF7, IRF-2 and higher levels of EBNA-1. In contrast in type I latency, low levels or absence of these negative factors plus potential positive regulator(s) presumably cause the activation of Qp. The primary activator(s) of Qp is still unknown (Figure 2). Some evidence suggests that Stats play a role in the activation of Qp, but the identity of the Stat(s) is uncertain. ${ }^{42}$ The biological significance of Qp inactivation in type III latency is unclear.

\section{IRF-7 as a regulator of the host immune system}

LMP-1 has been shown to regulate cellular immune genes including HLA 1, HLA II, Tap-1, and Tap-2. ${ }^{43,44}$ We examined the genomic sequence available in Genebank and found a putative ISRE sequence in the Tap-2 promoter region. Because LMP-1 induces Tap- 2 and IRF7, and IRF-7 has the potential to bind to Tap-2 ISRE, we reasoned that IRF-7 might be responsible for the induction of Tap-2 expression by LMP-1. The experimental results, summarized in the following, confirmed this hypothesis. Endogenous Tap-2 expression correlates with both IRF-7 and LMP-1 expression in different cell lines. LMP-1 induces Tap-2 expression in B cells in which IRF7 can be induced. Also, ectopic expression of IRF-7 enhances the induction of Tap- 2 by LMP-1. LMP-1 cannot induce Tap-2 in Akata Burkitt's lymphoma cells, in which
IRF-7 cannot be induced by LMP-1. However, the addition of IRF-7, which artificially restores the defect, activates the expression of Tap-2 in these cells. A Tap-2 promoter construct could be activated by IRF-7, and further enhanced by LMP-1 specifically. Additionally, the activation of the Tap-2 promoter was dependent on an intact ISRE sequence. Finally, IRF-7 could bind specifically to the ISRE in the Tap-2 promoter in vitro and to endogenous Tap-2 promoter sequence containing the ISRE in vivo under physiological conditions. ${ }^{32}$

In addition, it is apparent that IRF-7 is the most relevant IRF member for the activation of Tap-2 by LMP1 for the following reasons. Expression of IRF-2 is also associated with EBV type III latency, ${ }^{40}$ where levels of Tap-2 are high. IRF-2 could also bind to the Tap-2 ISRE as determined by EMSA. ${ }^{32}$ However, IRF-2 cannot activate the Tap-2 promoter reporter construct in at least two cell types. Nor could LMP-1 plus IRF-2 activate either the Tap-2 promoter reporter construct or endogenous Tap-2 in Akata cells in which IRF-7 is not inducible by LMP-1. ${ }^{32}$ Also, LMP-1 could not induce the expression of IRF-2 in several cell lines. ${ }^{30}$ Thus, IRF-2 appears not to be involved in the activation of Tap- 2 by LMP- 1 . Next, LMP-1 could specifically enhance the activation of the Tap-2 promoter produced by IRF-7, but not by IRF1 , although IRF-1 can activate the Tap- 2 promoter construct. Also, other IRFs tested, such as IRF-1, IRF-3, as well as ICSBP (IRF-8), are not associated with type III latency. ${ }^{30,40,41}$ Finally, LMP-1 facilitates the phosphorylation and nuclear translocation of IRF-7 for the activation of Tap-2. ${ }^{32}$

Since HLA 1, Tap-1 and Tap-2 are often induced simultaneously for antigen processing (e.g. by treatment with IFN- $\alpha$, IFN- $\gamma$, or LPS), and they all have potential binding sequence for IRF, it is possible that IRF-7 may also be involved in the regulation of Tap- 1 and HLA 1 genes. Current data suggest that this notion may be correct, and that IRF-7 may regulate both Tap-1 and HLA 1 in a fashion similar to Tap-2 (Zhang and Pagano, unpublished results). IRF-7 may thus be a key protein involved in the regulation of the immune system.

What advantage does EBV gain by inducing Tap-2 and other HLA 1-related genes? An LMP-1-expressing EBV latency state (Type III) is established transiently in primary infection of human B cells in vivo. ${ }^{1,2}$ These LMP-1-expressing cells have enhanced growth, survival and invasive potential, which allow the EBV-infected cells to proliferate quickly, thereby putting the human 
host at risk. This rapid proliferative process is checked by the appearance of EBV-specific primary cytotoxic $\mathrm{T}$ lymphocytes (CTL), which eliminate these type III latency cells because of the activation of Tap-2 and other HLA 1-related genes by LMP-1 and IRF-7, and ensure the safety of the host. In X-linked immunoproliferative disease in which $\mathrm{T}$ cell activation is defective,${ }^{45} \mathrm{EBV}$ infection is lethal. Interestingly, EBV in normal hosts still survives the CTL-attack by a transition to a type-I-like latency state, in which LMP-1 is not expressed. This type-I-like state, sometimes termed type 0 latency, can escape host immune surveillance, which ensures the survival of the virus. ${ }^{46-50}$ Because the whole process may ensure the survival both of the host and of the virus, a life-long coexistence may thus be maintained between the human host and EBV. In support of such a notion, the LMP-1-positive immunoblastic B cell lymphomas of the immunosuppressed are highly susceptible to restoration of the patients' $\mathrm{T}$ cell function or to adoptive CTL therapy. ${ }^{45,51-53}$ Thus, transient up-regulation of immune genes by IRF-7 and LMP-1 may set in motion selection of the persistent latently infected cells that do not express LMP-1 and eliminate these dangerous transformation-fated cells, resulting in survival of both the host and the virus (Figure 3).

Other than facilitating such selection, the rapid amplification of type III latency cells would also increase the supply of cells available for the switch to persistent state (Figure 3). The type-I-like persistent EBV-positive cells, which are considered as memory B cell-like, usually do not replicate. The transient establishment of type III latency may be the major contributor to and replenisher of the pool of persistently infected cells.

\section{IRF-7 as a putative oncogene}

Of the many human viruses identified, only a few are so far considered oncogenic including EBV. The unique feature of EBV is its ability to immortalize and to transform primary cells in vitro. LMP-1 is the principal oncoprotein contributing to immortalization and transformation; during this process, EBV establishes latency concomitantly. In addition, LMP-1 is expressed in several EBV-associated tumors, such as NPC, AIDS-associated lymphoma, and post-transplantation lymphoproliferative disorders (PTLD). ${ }^{1,2}$

The linkage between IRFs and cancer was first suggested by the finding that IRF-1 might be involved in oncogenesis as a tumor suppressor. ${ }^{54-56}$ Presently, several lines of evidence indicate the involvement of IRFs in oncogenesis, and their potential role in human cancer has been suggested. Overexpression of IRF-2 or IRF4 causes oncogenic transformation in NIH $3 \mathrm{~T} 3$ cells as well as in mice. ${ }^{56-58}$ Interestingly, some patients with multiple myeloma have a specific chromosomal translocation resulting in overexpression of IRF-4. This translocation is likely to be involved in the oncogenesis of this neoplasm. ${ }^{58}$ In addition, human herpes virus 8 (HHV8), also called Kaposi sarcoma associated herpes virus (KSHV), encodes several IRF-like molecules with partial homology to cellular IRFs (vIRFs). ${ }^{59}$ At least vIRF1 causes oncogenic transformation in both cell lines and mice. $^{60}$

With regards to IRF-7, available evidence suggests that IRF-7 may be oncogene-like and involved in the pathogenic processes triggered by EBV: first, expression and activation of IRF-7 protein are associated with the transformation state of EBV infection (type III latency). Second, LMP-1, the principal oncogenic protein required for EBV transformation, regulates IRF-7 at both transcriptional and post-translational levels. Third, the LMP-1 signaling pathways that induce IRF-7 largely parallel those used by LMP-1 to transform cells. Fourth, TPA (phorbol ester), a carcinogen, induces the expression of IRF-7 (Zhang and Pagano, unpublished results). In addition to the circumstantial evidence, IRF-7 itself, in the absence of LMP-1 or TPA, causes focus formation as well as anchorage-independent growth in NIH 3 T3 cells (Zhang, Der, and Pagano, unpublished results). Thus, it seems likely that IRF-7 may be an oncogene and intimately involved in the pathogenesis of EBV-associated tumors. More work is needed to address this issue.

\section{Concluding remarks}

Considering all the existing data, it is apparent that a novel signal transduction pathway has been discovered. That is, LMP-1 induces the expression of IRF-7, and further activates IRF-7 protein by phosphorylation and nuclear translocation post-translationally. Finally, activated IRF-7 mediates the regulation of EBV target genes involved in latency, immune regulation, and transformation (Figure 4). The interaction between LMP-1 and IRF-7 appears to be a triumph of natural selection and adaptation in viral evolution. 


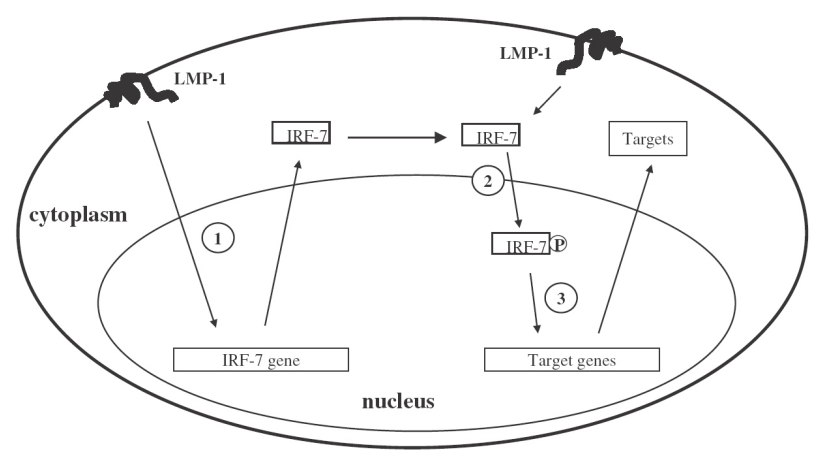

Figure 4. Relation between IRF-7 and LMP-1. (Step 1) LMP1 induces the expression of IRF-7. (Step 2) LMP-1 facilitates the phosphorylation and nuclear translocation of IRF-7. (Step 3) The activated IRF-7 mediates the regulation of target genes.

In addition to EBV, IRFs are likely to play important roles in other herpes viruses: infection by herpes simplex virus 1 (HSV-1) or human cytomegalovirus (HCMV) induces the expression of IRF-7 (Zhang and Pagano, unpublished results). HCMV activates IRF-3 protein. ${ }^{61}$ HHV-8 encodes four and rhesus rhadinovirus (RRV), encodes eight putative IRF homologues..$^{59,62}$ The functions of these viral IRFs need to be identified. It is apparent that IRFs hold key information for virus-cell interactions, the understanding of which will be essential for efficient treatment and preventive measures against viral diseases. The function of IRF-7 has also been extensively studied in the activation of virally induced expression of IFNs. Viral infection can activate IRF-7 by phosphorylation and nuclear translocation that are required for the activation of a subset of IFN genes. ${ }^{14,15,17-19}$ Also, IRF-7 may be one of the common targets attacked by different viruses, e.g. vaccinia virus E3L, HHV-8 vIRF-3, and influenza virus NS1 genes are all able to block activation of IRF-7 protein. ${ }^{63,64}$ Thus, IRF-7 likely plays an important role in cellular defense mechanisms against viruses.

In summary, research on IRF-7 has entered a new stage. IRF-7 appears to be a multi-functional protein, and plays an essential role in EBV latency and transformation. EBV latency may prove to be an invaluable system to decipher the biological roles of IRF-7 and serve as a model system for virus-IRF interaction.

\section{Acknowledgements}

We thank Dr Shannon Kenney for critical reading of the manuscript. This work was supported in part by grants from the National Institute of Allergy and Infectious Diseases (AI 42372-01) and from the National Cancer Institute (CA 19014).

\section{References}

1. A B Rickinson and E Kieff, Epstein-Barr virus. In: B N Fields, D M Knipe, and P M Howley, Editors, Virology, Lippinscott-Raven Publishers, Philadelphia, PA (1996), pp. 2397-2446.

2. E Kieff, Epstein-Barr virus and its replication. In: B N Fields, D M Knipe, and P M Howley, Editors, Virology, Lippinscott-Raven Publishers, Philadelphia, PA (1996), pp. 2343-2396.

3. M Miyamoto, T Fujita, Y Kimura, M Maruyama, H Harada, Y Sudo, T Miyata, and T Taniguchi, Regulated expression of a gene encoding a nuclear factor, IRF-1, that specifically binds to IFNbeta gene regulatory elements. Cell 54 (1988), pp. 903-913.

4. N Tanaka and T Taniguchi, The interferon regulatory factors and oncogenesis. Semin Cancer Biol 10 (2000), pp. 73-81.

5. H Nguyen, J Hiscott, and P M Pitha, The growing family of interferon regulatory factors. Cytokine Growth Factor Rev 8 (1997), pp. 293-312.

6. C R Escalante, J Yie, D Thanos, and A K Aggarwal, Structure of IRF-1 with bound DNA reveals determinants of interferon regulation. Nature 391 (1998), pp. 103-106.

7. N Tanaka, T Kawakami, and T Taniguchi, Recognition DNA sequences of interferon regulatory factor 1 (IRF-1) and IRF-2, regulators of cell growth and the interferon system. Mol Cell Biol 13 (1993), pp. 4531-4538.

8. J E J Darnell, I M Kerr, and G R Stark, Jak-STAT pathways and transcriptional activation in response to IFNs and other extracellular signaling proteins. Science 264 (1994), pp. 1415-1421.

9. P H Driggers, D L Ennist, S L Gleason, W H Mak, M S Marks, B Z Levi, J R Flanagan, E Appella, and K Ozato, An interferon gamma-regulated protein that binds the interferon-inducible enhancer element of major histocompatibility complex class I genes. Proc Natl Acad Sci USA 87 (1990), pp. 3743-3747.

10. S Goodbourn and T Maniatis, Overlapping positive and negative regulatory domains of the human beta-interferon gene. Proc Natl Acad Sci USA 85 (1988), pp. 1447-1451.

11. S Goodbourn, K Zinn, and T Maniatis, Human beta-interferon gene expression is regulated by an inducible enhancer element. Cell 41 (1985), pp. 509-520.

12. L Zhang and J S Pagano, IRF-7, a new interferon regulatory factor associated with Epstein-Barr virus latency. Mol Cell Biol 17 (1997), pp. 5748-5757.

13. T Durfee, K Becherer, P L Chen, S H Yeh, Y Yang, A E Kilburn, W H Lee, and S J Elledge, The retinoblastoma protein associates with the protein phosphatase type 1 catalytic subunit. Genes Dev 7 (1993), pp. 555-569.

14. W C Au, P A Moore, D W LaFleur, B Tombal, and P M Pitha, Characterization of the interferon regulatory factor-7 and its potential role in the transcription activation of interferon A genes. $J$ Biol Chem 273 (1998), pp. 29210-29217.

15. M G Wathelet, C H Lin, B S Parekh, L V Ronco, P M Howley, and T Maniatis, Virus infection induces the assembly of coordinately activated transcription factors on the IFN-beta enhancer in vivo. Mol Cell 1 (1998), pp. 507-518. 
16. R Lin, P Genin, Y Mamane, and J Hiscott, Selective DNA binding and association with the CREB binding protein coactivator contribute to differential activation of alpha/beta interferon genes by interferon regulatory factors 3 and 7. Mol Cell Biol 20 (2000), pp. 6342-6353.

17. R Lin, Y Mamane, and J Hiscott, Multiple regulatory domains control IRF-7 activity in response to virus infection. $J$ Biol Chem 275 (2000), pp. 34320-34327.

18. M Sato, et al., Distinct and essential roles of transcription factors IRF-3 and IRF-7 in response to viruses for IFN-alpha/beta gene induction. Immunity 13 (2000), pp. 539-548.

19. I Marie, J E Durbin, and D E Levy, Differential viral induction of distinctinterferon-alpha genes by positive feedback through interferon regulatory factor-7. EMBO J 17 (1998), pp. 6660-6669.

20. O Gires, U Zimber-Strobl, R Gonnella, M Ueffing, G Marschall, R Zeidler, D Pich, and W Hammerschmidt, Latent membrane protein 1 of Epstein-Barr virus mimics a constitutively active receptor molecule. EMBO J 16 (1997), pp. 6131-6140.

21. D Liebowitz, D Wang, and E Kieff, Orientation and patching of the latent infection membrane protein encoded by Epstein-Barr virus. J Virol 58 (1986), pp. 233-237.

22. J E Floettmann and M Rowe, Epstein-Barr virus latent membrane protein-1 (LMP1) C-terminus activation region 2 (CTAR2) maps to the far C-terminus and requires oligomerization for NF$\kappa \mathrm{B}$ activation. Oncogene 15 (1997), pp. 1851-1858.

23. S Murono, H Inoue, T Tanabe, I Joab, T Yoshizaki, M Furukawa, and J S Pagano, Induction of cycloxygenase-2 by Epstein-Barr virus latent membrane protein 1 is involved in vascular endothelial growth factor production in nasopharyngeal carcinoma cells. Proc Natl Acad Sci USA (in press) (2001).

24. K L Fries, W E Miller, and N Raab-Traub, Epstein-Barr virus latent membrane protein 1 blocks p53-mediated apoptosis through the induction of the A20 gene. J Virol 70 (1996), pp. 8653-8659.

25. W E Miller, H S Earp, and N Raab-Traub, The Epstein-Barr virus latent membrane protein 1 induces expression of the epidermal growth factor receptor. J Virol 69 (1995), pp. 4390-4398.

26. D Wang, D Leibowitz, and E Kieff, An EBV membrane protein expressed in immortalized lymphocytes transforms established rodent cells. Cell 43 (1985), pp. 831-840.

27. F Wang, C Gregory, C Sample, M Rowe, D Liebowitz, R Murray, A Rickinson, and E Kieff, Epstein-Barr virus latent membrane protein (LMP1) and nuclear proteins 2 and $3 \mathrm{C}$ are effectors of phenotypic changes in B lymphocytes: EBNA-2 and LMP1 cooperatively induce CD23. J Virol 64 (1990), pp. 2309-2318.

28. T Yoshizaki, H Sato, M Furukawa, and J S Pagano, The expression of matrix metalloproteinase 9 is enhanced by Epstein-Barr virus latent membrane protein 1. Proc Natl Acad Sci USA 95 (1998), pp. 3621-3626.

29. A Calender, M Billaud, J P Aubry, J Banchereau, M Vuillaume, and $\mathrm{G}$ M Lenoir, Epstein-Barr virus (EBV) induces expression of B-cell activation markers on in vitro infection of EBV-negative B-lymphoma cells. Proc Natl Acad Sci USA 84 (1987), pp. 8060-8064.

30. L Zhang and J S Pagano, Interferon regulatory factor 7 is induced by Epstein-Barr virus latent membrane protein 1. J Virol 74 (2000), pp. 5748-5757.

31. L Zhang, L H Wu, and J S Pagano, Involvement of tumor necrosis factor receptor-associated factors and nuclear factor-kB in the induction of interferon regulatory factor 7 by Epstein-Barr virus latent membrane protein 1 (submitted).

32. L Zhang and J S Pagano, Interferon regulatory factor 7 mediates the activation of Tap-2 by Epstein-Barr virus latent membrane protein 1. J Virol 75 (2001), pp. 341-350.

33. T K Kim, T Kim, T Y Kim, W G Lee, and J Yim, Chemotherapeutic DNA-damaging drugs activate interferon regulatory factor-7 by the mitogen-activated protein kinase kinase-4-cJun NH2-terminal kinase pathway. Cancer Res 60 (2000), pp. 1153-1156.

34. Q Tao, K D Robertson, A Manns, A Hildesheim, and R F Ambinder, The Epstein-Barr virus major latent promoter Qp is constitutively active, hypomethylated, and methylation sensitive. $J$ Virol 72 (1998), pp. 7075-7083.

35. I K Ruf, A Moghaddam, F Wang, and J Sample, Mechanisms that regulate Epstein-Barr virus EBNA-1 gene transcription during restricted latency are conserved among lymphocryptoviruses of old world primates. J Virol 73 (1999), pp. 1980-1989.

36. J Sample, E Henson, and C Sample, The Epstein-Barr virus nuclear protein 1 promoter active in type I latency is autoregulated. J Virol 66 (1992), pp. 4654-4661.

37. N S Sung, J Wilson, and J S Pagano, Characterization of cis-acting elements of the BamHI-F promoter of EBV. In: T Tursz, J S Pagano, D V Ablashi, G de The, G Lenoir, and G R Pearson, editors, The Epstein-Barr Virus and Associated Diseases, INSERM/ John Libbey Eurotext Limited, London (1993).

38. N S Sung, J Wilson, M Davenport, N D Sista, and J S Pagano, Reciprocal regulation of the Epstein-Barr virus BamHI-F promoter by EBNA-1 and an E2F transcription factor. Mol and Cell Biol 14 (1994), pp. 7144-7152.

39. M Davenport and J S Pagano, Expression of EBNA-1 mRNA is regulated by cell-cycle during Epstein-Barr virus type I latency. J Virol 73 (1999), pp. 3154-3161.

40. L Zhang and J S Pagano, Interferon regulatory factor 2 represses the Epstein-Barr virus BamHI-Q latency promoter in type III Latency. Mol Cell Biol 19 (1999), pp. 3216-3223.

41. C Nonkwelo, I K Ruf, and J Sample, Interferon-independent and induced regulation of Epstein-Barr virus EBNA-1 gene transcription in Burkitt lymphoma. J Virol 71 (1997), pp. 6887-6897.

42. H Chen, J M Lee, Y Wang, D P Huang, R F Ambinder, and S D Hayward, The Epstein-Barr virus latency BamHI-Q promoter is positively regulated by STATs and Zta interference with JAK/ STAT activation leads to loss of BamHI-Q promoter activity. Proc Natl Acad Sci USA 96 (1999), pp. 9339-9344.

43. Q Zhang, L Brooks, P Busson, F Wang, D Charron, E Kieff, A B Rickinson, and T Tursz, Epstein-Barr virus (EBV) latent membrane protein 1 increases HLA class II expression in an EBVnegative B cell line. Eur J Immunol 24 (1994), pp. 1467-1470.

44. M Rowe, et al., Restoration of endogenous antigen processing in Burkitt's lymphoma cells by Epstein-Barr virus latent membrane protein-1: coordinate up-regulation of peptide transporters and HLA-class I antigen expression. Eur J Immunol 25 (1995), pp. 1374-1384.

45. J Sayos, et al., The X-linked lymphoproliferative-disease gene product SAP regulates signals induced through the co-receptor SLAM. Nature 395 (1998), pp. 462-469.

46. F Chen, J-Z Zou, L DiRenzo, G Winberg, L-F Hu, E Klein, G Klein, and I Ernberg, A subpopulation of normal B cells latently infected with Epstein-Barr virus resembles Burkitt lymphoma 
cells in expressing EBNA-1 but not EBNA-2 or LMP-1. $J$ Virol 69 (1995), pp. 3752-3758.

47. E M Miyashita, B Yang, G J Babcock, and D A Thorley-Lawson, Identification of the site of Epstein-Barr virus persistence in vivo as a resting B cell. $J$ Virol 71 (1997), pp. 4882-4891.

48. E M Miyashita and D A Thorley-Lawson, A new form of Epstein-Barr virus latency in vivo. Curr Top Microbiol Immunol 194 (1995), pp. 135-144.

49. L Qu and D T Rowe, Epstein-Barr virus latent gene expression in uncultured peripheral blood lymphocytes. J Virol 66 (1992), pp. 3715-3724.

50. R Tierney, N Steven, L Young, and A Rickinson, Epstein-Barr virus latency in blood mononuclear cells: analysis of viral gene transcription during primary infection and in the carrier state. $J$ Virol 68 (1994), pp. 7374-7378.

51. D H Crawford and J M Edwards, Immunity to Epstein-Barr virus in cyclosporin A-treated renal allograft recipients. Lancet $\mathbf{1}$ (1982), pp. 1469-1470.

52. D H Crawford, P Sweny, J M Edwards, G Janossy, and A V Hoffbrand, Long-term T-cell-mediated immunity to Epstein-Barr virus in renal-allograft recipients receiving cyclosporin A. Lancet 1 (1981), pp. 10-12.

53. C M Rooney, M A Roskrow, C A Smith, M K Brenner, and H E Heslop, Immunotherapy for Epstein-Barr virus-associated cancers. J Natl Cancer Inst Monogr 23 (1998), pp. 89-93.

54. N Tanaka, M Ishihara, and T Taniguchi, Suppression of c-myc or fosB-induced cell transformation by the transcription factor IRF1. Cancer Lett 83 (1994), pp. 191-196.)

55. N Tanaka, M Ishihara, M Kitagawa, H Harada, T Kimura, T Matsuyama, M S Lamphier, S Aizawa, T W Mak, and T Taniguchi, Cellular commitment to oncogene-induced transformation or apoptosis is dependent on the transcription factor IRF-1. Cell 77 (1994), pp. 829-839.

56. H Harada, M Kitagawa, N Tanaka, H Yamamoto, K Harada, M Ishihara, and $\mathrm{T}$ Taniguchi, Anti-oncogenic and oncogenic potentials of interferon regulatory factors-1 and -2. Science 259 (1993), pp. 971-974.
57. H Nguyen, A Mustafa, J Hiscott, and R Lin, Transcription factor IRF-2 exerts its oncogenic phenotype through the DNA binding/transcription repression domain. Oncogene 11 (1995), pp. $537-544$

58. S Iida, P H Rao, M Butler, P Corradini, M Boccadoro, B Klein, R S Chaganti, and R Dalla-Favera, Deregulation of MUM1/IRF4 by chromosomal translocation in multiplemyeloma. Nat Genet 17 (1997), pp. 226-230.

59. P M Pitha, W C Au, W Lowther, Y T Juang, S L Schafer, L Burysek, J Hiscott, and P A Moore, Role of the interferon regulatory factors (IRFs) in virus-mediated signaling and regulation of cell growth. Biochimie 80 (1998), pp. 651-658.

60. S J Gao, C Boshoff, S Jayachandra, R A Weiss, Y Chang, and P S Moore, KSHV ORF K9 (vIRF) is an oncogene which inhibits the interferon signaling pathway. Oncogene 15 (1997), pp. 1979-1985

61. L Navarro, K Mowen, S Rodems, B Weaver, N Reich, D Spector, and M David, Cytomegalovirus activates interferon immediateearly response gene expression and an interferon regulatory factor 3-containing interferon-stimulated response element-binding complex. Mol Cell Biol 18 (1998), pp. 3796-3802.

62. L Alexander, L Denekamp, A Knapp, M R Auerbach, B Damania, and R C Desrosiers, The primary sequence of rhesus monkey rhadinovirus isolate 26-95: sequence similarities to Kaposi's sarcoma-associated herpesvirus and rhesus monkey rhadinovirus isolate 17577. J Virol 74 (2000), pp. 3388-3398.

63. B Lubyova and P M Pitha, Characterization of a novel human herpesvirus 8-encoded protein, vIRF-3, that shows homology to viral and cellular interferon regulatory factors. J Virol 74 (2000), pp. 8194-8201.

64. E Smith, I Marie, A Prakash, A Garcia-Sastre, and D E Levy, IRF3 and IRF7 phosphorylation in virus-infected cells does not require double-stranded RNA-dependent protein kinase $\mathrm{R}$ or IkB kinase but is blocked by vaccinia virus E3L protein. $J$ Biol Chem 276 (2001), pp. 8951-8957. 